chlamydia and HSV). There were 2 new diagnoses of HIV at the time of GC diagnosis, and 2 further cases at 3 months followup.

Discussion/conclusion Management of rectal GC did not reach the BASHH targets on any recommendation, suggesting that improvements in managing rectal GC are needed within our clinic. Re-testing and re-attendance were poor. Staff has received further training and a re-audit in 2017 will assess improvement. We have established a robust call/recall system to enable early diagnosis of HIV which was significant in our cohort of men with rectal GC.

\section{P168 CHEMSEX: A HEALTH NEEDS ASSESSMENT FOR AN EMERGING PUBLIC HEALTH CONCERN}

Andrew Tomkins*, Roberto Vivancos, Merav Kliner. Public Health England, Greater Manchester, UK

\subsection{6/sextrans-2016-052718.218}

Background/introduction Reports of sexualised drug taking (chemsex) have increased significantly in recent years. To establish the risks associated with chemsex and the services required by participants in Greater Manchester, a health needs assessment was undertaken.

Aim(s)/objectives To identify links between chemsex and adverse health outcomes, and to determine the perceived barriers seeking support.

Methods An online survey was devised, and then promoted with the support of local voluntary organisations and sexual health clinics. Data were analysed on acute Hepatitis $\mathrm{C}$ diagnoses for the previous 5 years using data collected by PHE. Interviews were conducted with key stakeholders.

Results In total, 54 participants completed the anonymous online survey, of which 52 were men who have sex with men (MSM). $76 \%$ were HIV positive and 20\% were Hepatitis C positive. The most commonly used recreational drugs were Mephedrone (81\%) and GHB/GBL (79\%). Of respondents, 78\% felt they would prefer to access support in a specialist clinic within a sexual health service. Qualitative data on barriers to accessing support were determined. Using PHE Acute Hepatitis C data, 46\% of the 57 patients diagnosed via Greater Manchester sexual health clinics between 2009 - 2015 had used at least one chemsex drugs in the past 12 months. Stakeholder interviews gave insight into perceived barriers to accessing care.
Discussion/conclusion We identify demographic factors of chemsex users and the perceived barriers to accessing support. These findings will be useful in guiding commissioning and tailoring specialist services.

\section{P169 COMPARISON OF THE FTD ${ }^{\text {TM }}$ URETHRITIS PLUS (7-PLEX) DETECTION KIT WITH ROUTINE SEXUAL HEALTH CLINIC NUCLEIC ACID AMPLIFICATION TESTING FOR DETECTION OF NEISSERIA GONORRHOEAE AND CHLAMYDIA TRACHOMATIS IN URINE, VAGINAL, PHARYNGEAL AND RECTAL SAMPLES}

${ }^{1}$ Mark Harrison* 2,1 Emma Harding-Esch, ${ }^{1}$ Claire Broad ${ }^{1}$ Clare Soares, ${ }^{1}$ Sebastian Fuller, ${ }^{2}$ Sandra Okala, ${ }^{3}$ John Saunders, ${ }^{4}$ Tristan Barber, ${ }^{5}$ Phillip Hay, ${ }^{1,2}$ Syed Tariq Sadiq. ${ }^{1}$ St George's University of London, London, UK; ${ }^{2}$ Public Health England, London, UK; ${ }^{3}$ Central and North West London NHS Foundation Trust, London, UK; ${ }^{4}$ Chelsea and Westminster Hospital NHS Foundation Trust, London, UK; ${ }^{5}$ St George's University Hospitals NHS Foundation Trust, London, UK

\subsection{6/sextrans-2016-052718.219}

Background/introduction The FTD $^{\mathrm{TM}}$ Urethritis Plus (FTDU) nucleic acid amplification test (NAAT) detects seven pathogens associated with urethritis, including Chlamydia trachomatis (CT), Neisseria gonorrhoeae (NG), Mycoplasma genitalium, Trichomonas vaginalis, Mycoplasma hominis, Ureaplasma urealyticum and Ureaplasma parvum.

Aim(s)/objectives To perform an initial diagnostic evaluation of FTDU performance for NG and CT, compared to routine clinic NAAT (BD Viper), in prospectively collected genital samples from symptomatic patients.

Methods Alongside routine clinical samples, additional samples ( $\mathrm{n}=684$ ) were taken from symptomatic patients: females (vulvovaginal swabs; VVS), men-who-have-sex-with-women (MSW) (urine) and men-who-have-sex-with-men (MSM) (rectal and pharyngeal swabs; urine).

Results The prevalence of CT was 9.38\% across sample sites tested (24 Female, 21 Male, 3 MSM Urine, 1 MSM Pharynx and 5 MSM Rectal positives). The prevalence of NG was $9.74 \%$ across sample sites tested (5 Female, 6 Male, 10 MSM Urine, 17 MSM Pharynx and 19 MSM Rectal positives).

Discussion/conclusion FTDU was accurate for detecting CT from genital sites only and had poor sensitivity for NG at all sampling sites. This test could not be used for NG testing for urine or extra genital testing without supplementary testing according to the BASHH guidelines as the PPV is below 90\%.

Abstract P169 Table 1 FTDTM Urethritis Plus (7-Plex) detection kit

\begin{tabular}{|c|c|c|c|c|c|c|c|c|c|c|}
\hline & $\mathrm{CT}$ & & & & & NG & & & & \\
\hline Sample type (n) & Females (287) & Males (98) & $\begin{array}{l}\text { MSM Urine } \\
\text { (56) }\end{array}$ & $\begin{array}{l}\text { MSM Rectal } \\
\text { (67) }\end{array}$ & $\begin{array}{l}\text { MSM Pharynx } \\
\text { (71) }\end{array}$ & $\begin{array}{l}\text { Females } \\
(291)\end{array}$ & $\begin{array}{l}\text { Males } \\
(98)\end{array}$ & $\begin{array}{l}\text { MSM Urine } \\
\text { (57) }\end{array}$ & $\begin{array}{l}\text { MSM Rectal } \\
\text { (67) }\end{array}$ & $\begin{array}{l}\text { MSM Pharynx } \\
\text { (72) }\end{array}$ \\
\hline $\begin{array}{l}\text { Sensitivity } \% \\
\left(95 \% \mathrm{Cl}^{\mathrm{a}}\right)\end{array}$ & $\begin{array}{l}100 \\
\left(85.7-100^{b}\right)\end{array}$ & $\begin{array}{l}100 \\
\left(83.2-100^{b}\right)\end{array}$ & $\begin{array}{l}66.7 \\
(9.4-99.2)\end{array}$ & $\begin{array}{l}21.9 \\
(14.7-94.7)\end{array}$ & $\begin{array}{l}50.0 \\
(1.3-98.7)\end{array}$ & $\begin{array}{l}80.0 \\
(28.4-99.5)\end{array}$ & $\begin{array}{l}83.3 \\
(35.9-99.6)\end{array}$ & $\begin{array}{l}50.0 \\
(18.7-81.3)\end{array}$ & $\begin{array}{l}78.9 \\
(54.4-93.9)\end{array}$ & $\begin{array}{l}64.7 \\
(38.3-85.8)\end{array}$ \\
\hline $\begin{array}{l}\text { Specificity } \% \\
\left(95 \% \mathrm{Cl}^{\mathrm{a}}\right)\end{array}$ & $\begin{array}{l}99.6 \\
(97.8-100.0)\end{array}$ & $\begin{array}{l}97.4 \\
(90.9-99.7)\end{array}$ & $\begin{array}{l}98.1 \\
(89.9-100)\end{array}$ & $\begin{array}{l}96.8 \\
(88.8-99.6)\end{array}$ & $\begin{array}{l}100 \\
\left(94.4-100^{b}\right)\end{array}$ & $\begin{array}{l}99.6 \\
(98.0-100.0)\end{array}$ & $\begin{array}{l}100 \\
\left(96.1-100^{b}\right)\end{array}$ & $\begin{array}{l}100 \\
\left(92.3-100^{b}\right)\end{array}$ & $\begin{array}{l}83.3 \\
(69.8-92.5)\end{array}$ & $\begin{array}{l}96.4 \\
(87.5-99.6)\end{array}$ \\
\hline $\operatorname{PPV}\left(97.5 \% \mathrm{Cl}^{\mathrm{a}}\right)$ & $\begin{array}{l}96.0 \\
(79.6-99.9)\end{array}$ & $\begin{array}{l}91.3 \\
(71.9-98.9)\end{array}$ & $\begin{array}{l}66.7 \\
(9.4-99.2)\end{array}$ & $\begin{array}{l}21.9 \\
(14.8-94.7)\end{array}$ & $\begin{array}{l}100 \\
\left(2.5-100^{b}\right)\end{array}$ & $\begin{array}{l}80 \\
(28.4-99.5)\end{array}$ & $\begin{array}{l}100 \\
\left(47.8-100^{b}\right)\end{array}$ & $\begin{array}{l}100 \\
\left(47.8-100^{b}\right)\end{array}$ & $\begin{array}{l}65.2 \\
(42.7-83.6)\end{array}$ & $\begin{array}{l}84.6 \\
(54.6-98.1)\end{array}$ \\
\hline NPV $\left(95 \% \mathrm{Cl}^{\mathrm{a}}\right)$ & $\begin{array}{l}100 \\
\left(98.6-100^{\mathrm{b}}\right)\end{array}$ & $\begin{array}{l}100 \\
\left(95.2-100^{b}\right)\end{array}$ & $\begin{array}{l}98.1 \\
(89.9-100)\end{array}$ & $\begin{array}{l}96.8 \\
(88.8-99.6)\end{array}$ & $\begin{array}{l}97.2 \\
(90.2-99.7)\end{array}$ & $\begin{array}{l}99.6 \\
(98.0-100.0)\end{array}$ & $\begin{array}{l}98.9 \\
(94.1-100)\end{array}$ & $\begin{array}{l}90.2 \\
(78.6-96.7)\end{array}$ & $\begin{array}{l}90.9 \\
(78.3-97.5)\end{array}$ & $\begin{array}{l}89.8 \\
(79.2-96.2)\end{array}$ \\
\hline
\end{tabular}

